\title{
Comparative performance of OM-OFDM in broadband systems
}

\author{
K. Dhuness and B. T. Maharaj
}

\begin{abstract}
A method called offset modulation (OM-OFDM) is proposed to control the peak-to-average power ratio (PAPR) of an OFDM signal. The authors demonstrate the significant modulation, structural and performance differences between an OM-OFDM and CE-OFDM method. The OM-OFDM method in addition is able to accurately control the PAPR of a transmission for a targeted BER, which is currently not possible with CE-OFDM. By using a power performance decision metric (D), the OM-OFDM method is shown to offer a $34 \mathrm{~dB}$ and $3.44 \mathrm{~dB}$ net power performance gain (at a BER of $10^{-4}$ ) when compared to a CE-OFDM and traditional OFDM transmission for frequency selective fading channel conditions, respectively.
\end{abstract}

\section{INTRODUCTION}

A major disadvantage of orthogonal frequency division multiplexing (OFDM) is its high peak-toaverage power ratio (PAPR) [1]. This high PAPR reduces the battery life of a mobile device, which is not desirable. An offset modulation (OM-OFDM) method is proposed to control the PAPR of an OFDM transmission. The proposed OM-OFDM method may appear to be identical to phase modulation of an OFDM (CE-OFDM) transmission [2], but there are significant differences. The differences between an OM-OFDM and CE-OFDM method are evaluated in this letter and the benefits of the OM-OFDM method are presented.

\section{Modulation PROCESS}

A CE-OFDM transmission is ideally suited for constellations without imaginary components (e.g. BPSK). In cases where imaginary components exist (e.g. like in 16-QAM), as depicted in Fig. 1(a), this constellation is uniquely mapped onto a constellation without imaginary components (e.g. 16-QAM to 16-PAM mapping). This mapping process results in a severe bit error rate (BER) degradation. After the mapping process, depicted in Fig. 1(a), an inverse fast Fourier transform (IFFT) is performed on the 
mapped signal. The resultant OFDM signal denoted by $\phi(t)$ in Fig. 1(a), is phase modulated as shown below

$$
S(t)=A_{c} \cos \left(2 \pi f_{c} t+2 \pi h \phi(t)\right) .
$$

Here, $A_{c}$ is the signal amplitude, $f_{c}$ is the carrier frequency and $h$ denotes the modulation index. An OM-OFDM transmission, on the contrary, modulates constellation containing both real and imaginary components, without a mapping process [1]. The OM-OFDM method modulates the signal (Fig. 1(b)) as discussed below.

$$
\Phi_{1}(t)=\frac{\mathfrak{R}(m(t))}{\varsigma} \text { and } \Phi_{2}(t)=\frac{\mathfrak{I}(m(t))}{\varsigma}
$$

Where $m(t)$ denotes the complex OFDM signal, $\varsigma$ refers to a constant division term, $\mathfrak{R}$ and $\mathfrak{I}$ refer to the real and imaginary parts of an OFDM signal respectively. In addition, $\Phi_{1}(t)$ and $\Phi_{2}(t)$ represent the equivalent real and imaginary OFDM phase mapping. These, $\Phi_{1}(t)$ and $\Phi_{2}(t)$ terms may now be combined into a co-sinusoid as follows

$$
s(t)=\cos \left(2 \pi f_{c} t+\Phi_{1}(t)+\Psi_{o s}\right)-\cos \left(2 \pi f_{c} t+\Phi_{2}(t)\right)
$$

where, $\Psi_{o s}$ refers to an offset term. The $\Psi_{o s}$ and $\zeta$ terms ensure that the receiver can successfully detect the originally transmitted signal. The OM-OFDM transmission may appear to lose its attractive OFDM equalization properties. However, as depicted in Fig. 1(b) the OM-OFDM equalization process is identical to that employed in OFDM, but structurally different to CE-OFDM. During a CE-OFDM transmission a frequency-domain equalizer (FDE), depicted in Fig. 1(a) is used to mitigate the effects of a channel. The FDE extracts channel state information (CSI) from the prefix (which consists of a guard interval (GI) and pilot symbol), which are inserted between successive CE-OFDM blocks. The CE-OFDM equalization process requires additional overhead (prefix) and complexity (FDE) when compared to an OM-OFDM transmission.

\section{DECISION METRIC}

A power performance decision metric [1] has been proposed to determine the net power performance gain offered by an OM-OFDM transmission. The decision metric is given by $D=\frac{E_{t}}{N_{o}} \cdot \frac{W}{R_{b}}$, where $N_{o}$ is the noise power, $W$ refers to the bandwidth occupancy and $R_{b}$ is the data rate. Furthermore, $E_{t}$ is the total energy per bit and can be written as $E_{t}=E_{b}+E_{w}$, where $E_{b}$ is the received energy per bit and $E_{w}$, is the wasted energy per bit due to inefficient power amplifier utilization. In order to determine $E_{t}$, the 


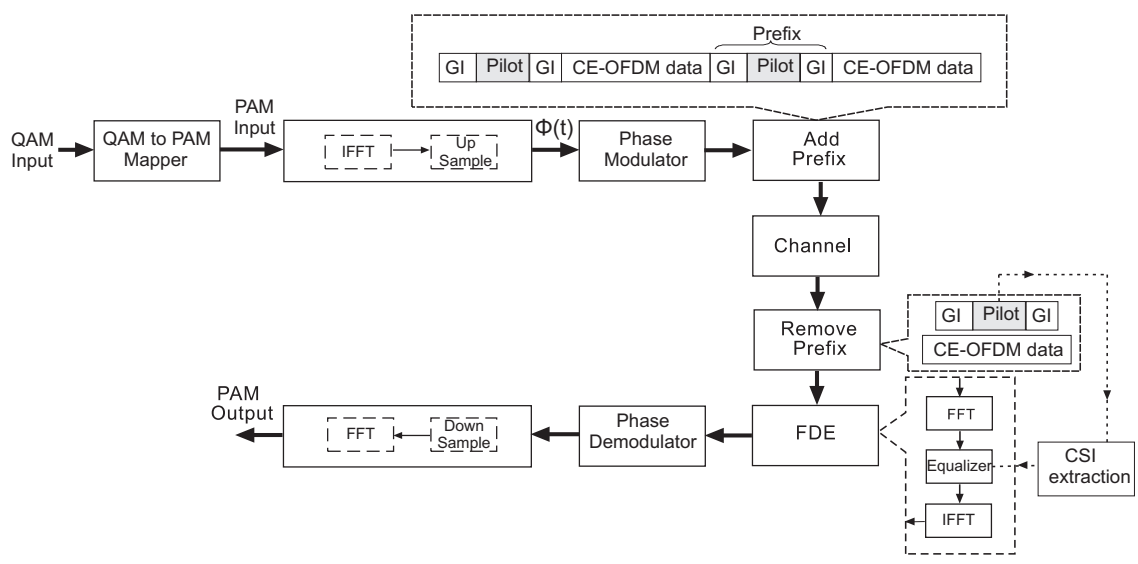

(a) CE-OFDM structure

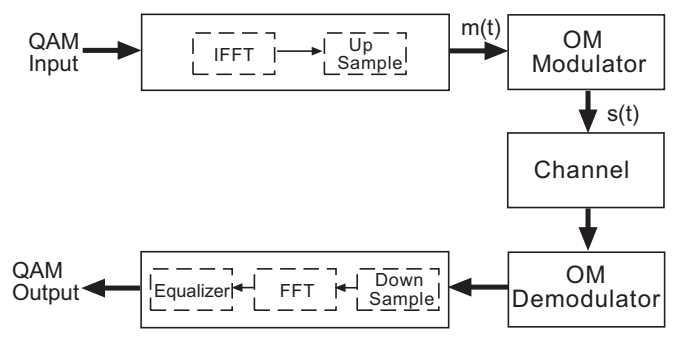

(b) OM-OFDM structure

Fig. 1. Transmitter-receiver structure

power added efficiency (PAE) of a standard off-the-shelf AN10858 RF power amplifier [3], which has been recommended for DVB-T [4] applications is used here for performance comparisons. A $2^{\text {nd }}$ degree polynomial was used to describe the PAE for this particular amplifier.

\section{RESULTS AND DISCUSSION}

In all the results which follow, the $2 \mathrm{k}$ mode of the DVB - T2 standard [4] was used to transmit OFDM, OM-OFDM and CE-OFDM (16-QAM Gray-coded) data through a 3-tap bad-urban frequency selective fading (FSF) channel [5]. Identical throughput and bandwidth occupancies (8 MHz) were used for all three methods. The simulation parameters used for OM-OFDM are described in [1], also $2 \pi \mathrm{h}=0.0628$ for a CE-OFDM transmission, while perfect carrier and timing synchronization is assumed. The averaged PAPR for both OFDM and CE-OFDM transmissions, are $12 \mathrm{~dB}$ and $3 \mathrm{~dB}$ respectively, when using the DVB-T2 standard. Hence from a direct comparison between OM-OFDM and OFDM (at a PAPR of $12 \mathrm{~dB}$ ) depicted in Fig. 2, it is noted that OM-OFDM offers similar BER characteristics as an OFDM transmission. At a BER of $10^{-4}$ the signal-to-noise ratio (SNR) of a CE-OFDM transmission is $68.85 \mathrm{~dB}$. This indicates the extent of the large power requirement needed for a fixed PAPR of $3 \mathrm{~dB}$. These PAPR's 


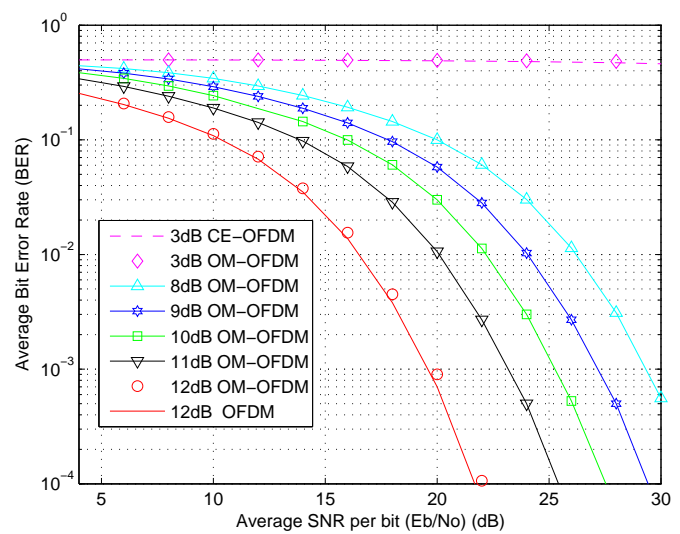

Fig. 2. A BER comparison between an OM-OFDM, OFDM and a CE-OFDM transmission in a 3-tap bad-urban FSF channel for various PAPR's.

are fixed for both OFDM and CE-OFDM, whereas OM-OFDM allows the designer to vary the PAPR for a desired BER, while still maintaining identical throughput and bandwidth occupancy as an OFDM or CE-OFDM transmission.

In order to facilitate a direct comparison between OFDM, OM-OFDM and a CE-OFDM transmission, the decision metric was employed. The results from this metric depicted in Fig. 3, indicate that the

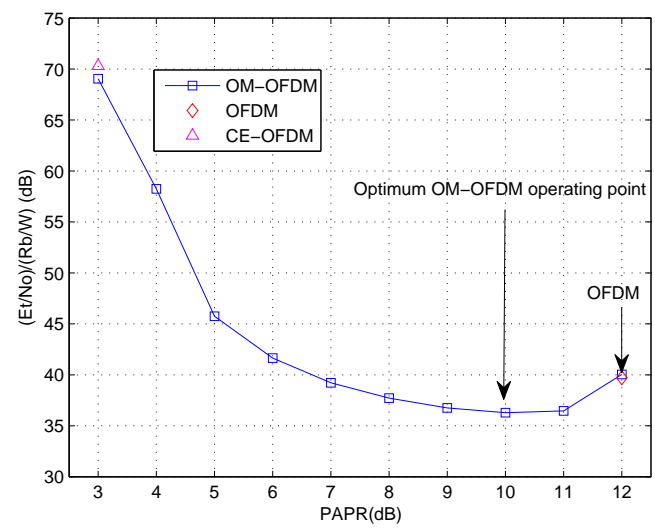

Fig. 3. A power performance comparison at a BER of $10^{-4}$ for an AN10858 amplifier.

optimum operating point for an OM-OFDM transmission is at a PAPR of $10 \mathrm{~dB}$ (where a minimum decision metric occurs). At this optimum operating point the OM-OFDM transmission is shown to offer a $34 \mathrm{~dB}$ and $3.44 \mathrm{~dB}$, net power performance gain (at a BER of $10^{-4}$ ) when compared to a CE-OFDM and OFDM transmission, respectively. Furthermore the decision metric suggests that the OM-OFDM 
method's average PAPR value may be lowered to $7 \mathrm{~dB}$ (thus a $5 \mathrm{~dB}$ average PAPR reduction), while still maintaining a performance improvement when compared to an OFDM transmission. This decision metric result might appear to be misleading since at a BER of $10^{-4}$, in Fig. 2, a $3.44 \mathrm{~dB}$ net gain is not expected. This net power performance gain is attributed to the fact that there is an exponential relationship between PAPR (dB) and PAE, instead of a linear relationship.

\section{CONCLUSiON}

The authors have shown that the proposed OM-OFDM method is significantly different to a conventional CE-OFDM transmission. By using a decision metric, OM-OFDM is shown to offer significant improvement when compared to both OFDM and CE-OFDM transmissions.

\section{REFERENCES}

[1] Dhuness K, Maharaj BTJ. An Offset Modulation scheme to control the PAPR of an OFDM transmission. In: Proceedings of the IEEE 72nd Vehicular Technology Conference. Ottawa, Canada; 2010. p. 1-5.

[2] Thompson SC. Constant Envelope Phase Modulation [PhD Thesis]. University of California. San Diego; 2005.

[3] Semiconductors N; 2010. AN10858.pdf. Available from: http://www.nxp.com/documents/application_note/.

[4] ETSI EN 302 755. Digital Video Broadcasting (DVB); 2009. Doc. 302.

[5] Patzold M. In: Mobile Fading Channels. John Wiley and Son; 2002. .

\section{AUTHORS AFFILIATION}

The authors are from the Department of Electrical, Electronic and Computer Engineering, University of Pretoria, Pretoria, 0002, South Africa e-mail: kdhuness@ieee.org. 